\title{
ENHANCED CENTRAL SYSTEM OF THE TRAVERSING ROD FOR HIGH-PERFORMANCE ROTOR SPINNING MACHINES
}

\author{
Jan Valtera, Petr Žabka and Jaroslav Beran
}

Technical University of Liberec, Faculty of Mechanical Engineering, Department of Textile Machine Design, Studentska 2, 46117 Liberec, Czech Republic, jan.valtera@tul.cz, jaroslav.beran@tul.cz +420 485353 158, +420 485353171

\begin{abstract}
:
The paper deals with the improvement of central traversing system on rotor spinning machines, where rectilinear motion with variable stroke is used. A new system of traversing rod with implemented set of magnetic-mechanical energy accumulators is described. Mathematical model of this system is analysed in the MSC. Software Adams/View and verified by an experimental measurement on a real-length testing rig. Analysis results prove the enhancement of devised traversing system, where the overall dynamic force is reduced considerably. At the same time, the precision of the traversing movement over the machine length is increased. This enables to increase machine operating speed while satisfying both the maximal tensile strength of the traversing rod and also output bobbin size standards. The usage of the developed mathematical model for determination of the optimal number and distribution of accumulators over the traversing rod of optional parameters is proved. The potential of the devised system for high-performance rotor spinning machines with longer traversing rod is also discussed.
\end{abstract}

\section{Keywords:}

rotor spinning machine, traversing rod, rectilinear motion, variable stroke, energy accumulation

\section{Introduction}

From the very beginning of the industrial production of rotor spinning machines, a modular concept with one central drive unit was introduced. With respect to the required number of sections, the rotor spinning machine is assembled by the connection of sections. In this arrangement all drives are distributed from central drive unit, which is placed at one machine end. Despite several new approaches, for example, introducing the machine with individually driven spinning units, the central motion distribution still represents the most economically effective system for rotor spinning machines. The nowadays rotor spinning machines are designed in length over $50 \mathrm{~m}$. According to the set trend, further increase in the length and the operating speed on the modern machines is required. This brings heavy demands on the moving elements, expeciallyon the central system of traversing rod [1]. Solutions enabling further enhancement of such system, for example, the accumulation of kinetic energy at the reversal points [2, 3, 4], are therefore vital for introducing high-performance rotor spinning machines with the central system of the traversing rod.

Each operation section consists of a certain number of spinning units forming a line within the section. Generally, the working unit consists of the input roving material compartment, rotor spinning unit with accessories and the yarn winding system [5]. In order to wind up the yarn into the output cross-wound packages, the yarn needs to be guided over the bobbin width during the winding process. This specific rectilinear movement is provided by yarn guides fixed to a traversing rod. The traversing rod with an overall length of the machine consists of assembled one-section-long rods of different material properties [1, 3]. Commonly, the traversing rod has the tubular profile with an outer diameter of $12 \mathrm{~mm}$. The traversing rod is connected to the machine frame by means of slide-free joints. At the same time, the one end of the rod is connected to the traversing mechanism, placed in the central drive unit. There are several types of traversing mechanisms [6]. Their function is to generate the required rectilinear motion.

The displacement of the traversing movement is shown in figure 1. The stroke characteristic consists of linear and transition section [7]. The transition section is described by the virtual cam transition angle $\mathrm{\gamma}$. This parameter reflects the necessary virtual cam angle, or corresponding width of the stroke, where the rod is decelerated (in the direction towards the reversal point) and accelerated (in the direction from the reversal point). The standard value of the transition angle $y$ varies from $16-32^{\circ}$, (approx. 9-18 $\mathrm{mm}$ ). The level of acceleration affects the overall dynamic forces upon the both traversing rod and the traversing mechanism, as shown in figure 2. While the higher value of $\mathrm{y}$ positively reduces the level of acceleration and therefore dynamic forces, it negatively increases the amount of yarn stored at the edges of the bobbin (bobbin flanges). This may lead to an insufficient structure of the output package. In order to meet the winding properties requirement with traversing movement of non-zero transition width, the additional motion, called reversal point shift is introduced. The stroke modification due to the reversal point shifting is shown in figure 1 , where 
the red and blue courses reflect the boundary stages. This provides the reversal points of the movement to be shifted within several millimetres. This additional movement, typically based on harmonic function with the amplitude of $2 \mathrm{~mm}$, is relatively slow and does not affect the dynamic behaviour of the system significantly. Generally, there are two types of the reversal point shifting: the conventional one (figure 1 a) and the variable one (figure $1 \mathrm{~b}$ ), [5, 8]. In both cases, the reversal point of the movement is slowly shifted from the inner reversal point $(I R P)$ to outer one $(O R P)$ and vice versa.

Figure 2 shows the theoretical dynamic force of the rigid traversing rod. The real force characteristic is further affected by the effect of the transverse and longitudinal vibration of the rod [9]. Excitation of this vibration relates to the transition function of the generated motion and also to clearances in joints and traversing mechanism parts. Despite using advanced rod materials on nowadays machines [1, 3], the overall mass can reach up to $8 \mathrm{~kg}$. At high speeds, the overall dynamic force can reach the maximal allowed tensile force $1000 \mathrm{~N}$.

As a result, the enormous forces exerted on the traversing system, limit its application in the high-performance machines. There have been several new approaches introduced in recent years [10,11], where the usage of individual drives for generating the traversing movement for each unit, is the most promising one [12]. However, it still represents a more expensive solution in the rotor spinning machinery.
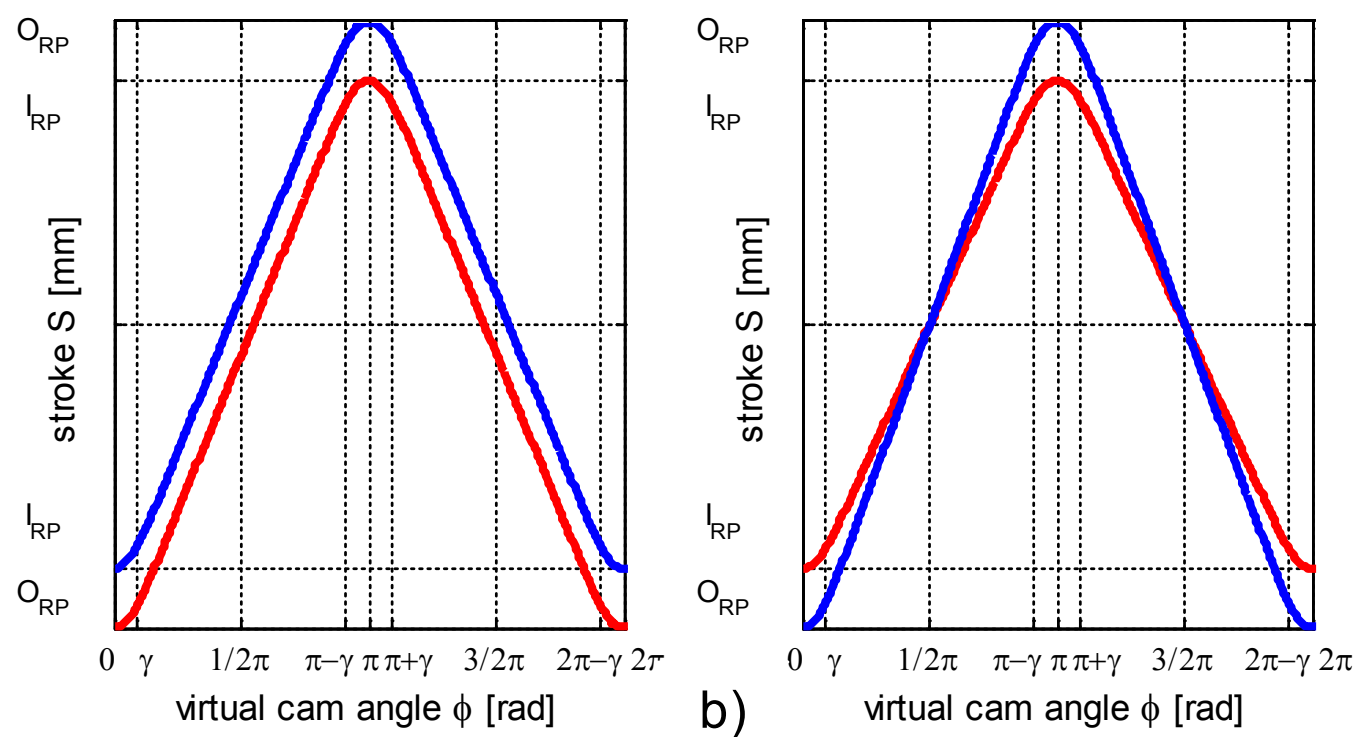

Figure 1. Reciprocating traversing motion characteristic with respect to virtual cam angle $\Phi$. Stroke modification due to the reversal point shifting (from ORP to IRP and vice versa). (a) Conventional shifting of the reversal point; (b) Variable shifting of the reversal point.

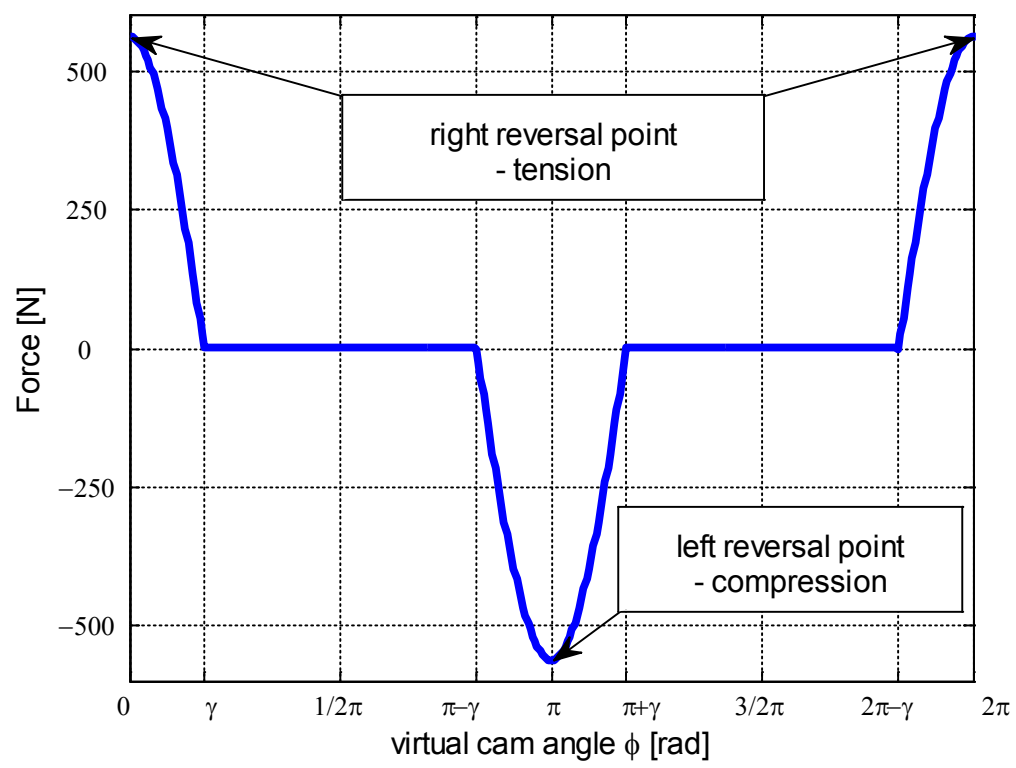

Figure 2. The force exerted on the traversing rod at the connecting point to the traversing mechanism. (The rigid traversing rod, at the traversing speed 200 RPM, $\mathrm{y}=32^{\circ}$ ). 
Another approach is based on the accumulation of the kinetic energy at the motion reversal points and thus, reduction of the dynamic forces upon the traversing elements. Several systems based either on mechanical spring [13] or on them agneticelementswithaddititionalservo-drvien support $[4,14]$ have been developed. With introducing of new highly effective magnetic compounds, e.g. NdFeB magnets, magnetic systems became more efficient and therefore widely used in industry. Based on the previous research of the traversing system, the new magnetic-mechanical accumulator of kinetic energy was invented at the Technical University of Liberec and described in $[15,2]$. This system uses the repelling force of strong permanent magnets in combination with a preloaded mechanical spring. The force characteristic with respect to the rod movement is shown in figure 3 .

In this system, the magnet that is fixed to the traversing rod is approaching towards oppositely magnetized magnet of the accumulator that is fixed to the preloaded spring. While the traversing rod moves towards the reversal point, the magnetic repelling force increases progressively. After reaching a certain preload of the spring, the magnet of the accumulator starts moving and therefore, the progressive force characteristic is replaced with the linear one. Implementing of the spring linear characteristic into the magnet progressive one enables to eliminate the maximal force difference at the reversal points of the movement with reversal point shifting. This system of noncontacting kinetic energy accumulation represents a reliable solution for the rotor spinning machine with the central traversing system as it does not require any additional drives and may be implemented into the nowadays machines without serious design modification. Because the accumulator consists of inner moveable parts, detrimental vibration effects appear during the operation. Despite a low impact of these vibration on the traversing rod, the system is beneficial especially for longer machines operating at lower speeds.

\section{Mathematical model of the traversing rod}

In order to analyse the system of traversing rod, the mathematical model of the system in the MSC. Software Adams/View was created [16] (hereinafter referred to as 'the original model'). The mathematical description is based on the discretization of the traversing rod into a set of masselements. Dynamic behaviour of the original model is defined by the linear differential equation (1).

$$
\mathbf{M} \cdot \ddot{\mathbf{x}}+\mathbf{B} \cdot \dot{\mathbf{x}}+\mathbf{K} \cdot \mathbf{x}+\mathbf{F}_{\mathbf{v}}=\mathbf{0}
$$

where $M$ is lumped mass matrix, $B$ is the material damping matrix, $K$ is the stiffness matrix, $x$ is the displacement vector and $F V$ is the vector of external forces including the passive resistance. The model element has 6 DOF. The extended definition of beam element in the software Adams/View is described in [17]. Structure of such a system is shown in figure 4. The model corresponding to the machine with 20 working sections is described by 320 elements with appropriate material properties and connections. Selected elements are connected to the ground by means of sliding joints with defined friction parameters. The real joints on the machine, however, include a certain clearance in the radial direction. Because of the major dynamic behaviour being present in the axial direction, these effects were neglected in the model. According to the position of sliding joints on the machine, linear joints with experimentally determined friction coefficient $f$ were imposed on the corresponding rod elements in the model. Material properties were obtained experimentally. The coefficient of viscous damping $b$ was evaluated by means of half-power bandwidth method $[1,3]$. The stiffness of the rod $k$ was determined from rod samples that have been measured on a universal tester.

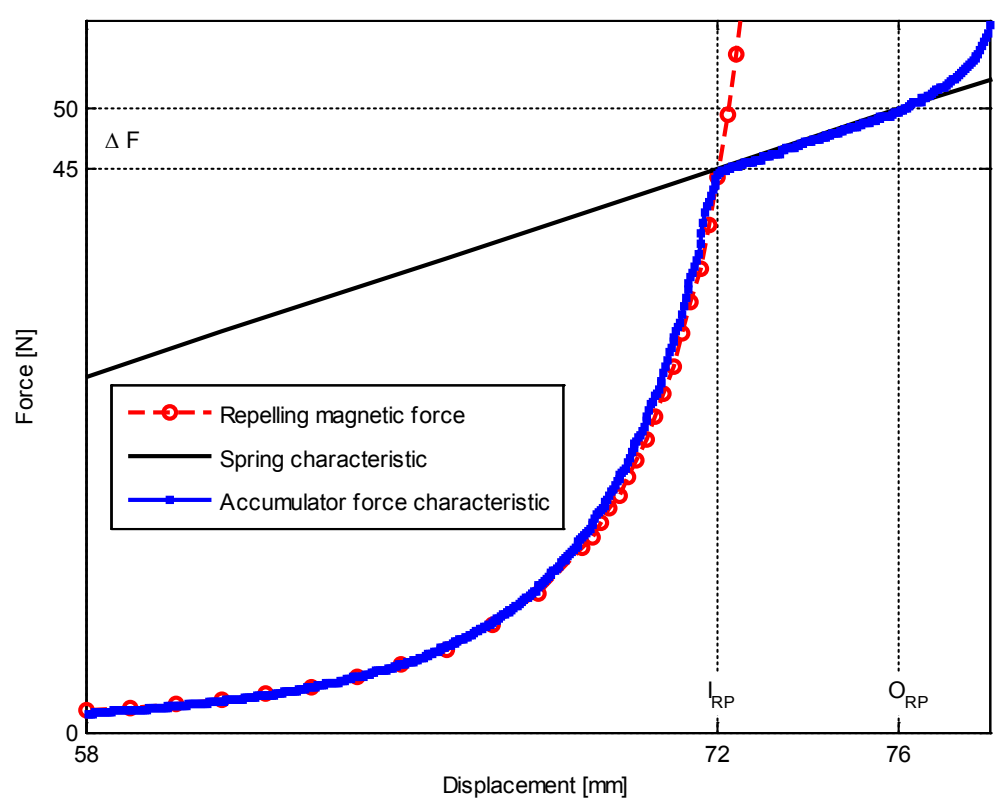

Figure 3. The force characteristic with respect to traversing movement (detailed view of the right reversal point area) of the developed magneticmechanical accumulator. 
The set boundary condition of the defined course of acceleration on the first element describes the rigid connection of the traversing rod to the mechanism generating the required traversing motion. The initial position of the system is set to the reversal point of the movement. It is difficult to set the initial velocity values of all model elements due to the elastic mechanical properties of the system. In order to avoid the potential discrepancy in the initial time of dynamic analysis, further periods of the movement have been evaluated. All the elements have its coordinate systems enabling to record kinematic and dynamic quantities. Primarily, the results on elements in the middle of $1^{\text {st }}, 5^{\text {th }}, 10^{\text {th }}, 15^{\text {th }}$ and $20^{\text {th }}$ sections have been evaluated.

In order to describe also the system with magnetic-mechanical accumulators the original model has been modified. The onesection length model with detailed accumulator definition, described in [2], uses both the magnetic parts and the preloaded spring to define the system behaviour. This description requires contact implementation and thus increases computing time considerably. For the full-length model with 20 -sections and 20 magnetic-mechanical accumulators (hereinafter referred to as "the modified model“), a simplified approach was used instead. This simplification is based on the assumption, that the kinetic energy of the decelerating rod is reduced substantially before the magnetic force reaches the spring pretension. In the less convenient case of $\gamma=16^{\circ}$, or corresponding stroke width of $9 \mathrm{~mm}$, the velocity is decreased by more than $50 \%$ before the spring became to be slightly pushed. The influence of the dumped vibration of the low-mass spring over the traversing rod is in this case relatively marginal and could be therefore neglected. Based on previous analyses and experiments, the influence of magnetic damping was also neglected, because of model and test results correspondence. However, for the more precise model definition, the magnetic damping could be added.

In the simplified model each accumulator is described by acting force $F m$ with respect to the measured position of appropriate element of the rod $x i$. The linear differential equation (1) is then modified to nonlinear by adding the vector of external forces $F m(x)$, see equation (2). This vector covers the external magnetic force $F m(x)$ exerted upon the particular element in the middle of each section.

$$
\mathbf{M} \cdot \ddot{\mathbf{x}}+\mathbf{B} \cdot \dot{\mathbf{x}}+\mathbf{K} \cdot \mathbf{x}+\mathbf{F}_{\mathbf{v}}+\mathbf{F}_{\mathbf{m}}(\mathbf{x})=\mathbf{0},
$$

The $F m(x)$ characteristic was defined in the model by means of imported data according the measured characteristic shown in figure 3. Furthermore, there are mass points connected to these elements, reflecting the additional mass of magnets on the rod $\mathrm{mm}$. The modification of the former system is redhighlighted in figure 4.

\section{Analysis and testing}

The dynamic analysis of the original model has been performed for a wide range of traversing speeds. Resulted quantities are presented with respect to the virtual cam angle and angular velocity. Figure 5 shows the dynamic

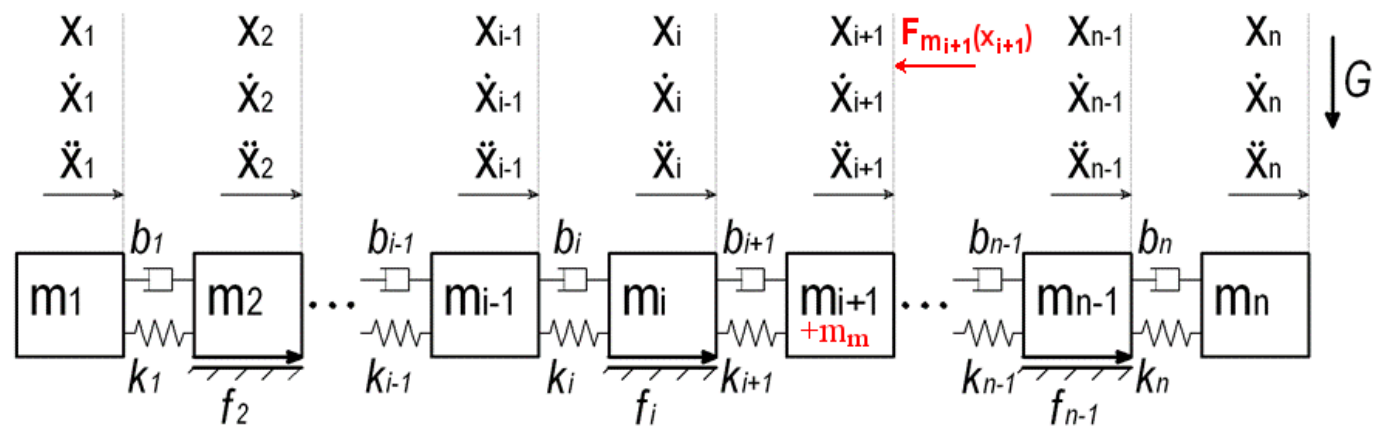

Figure 4.Schematic structure of mathematical model of the traversing system. (The model modification for the system with magnetic-mechanical accumulators is highlighted in red).

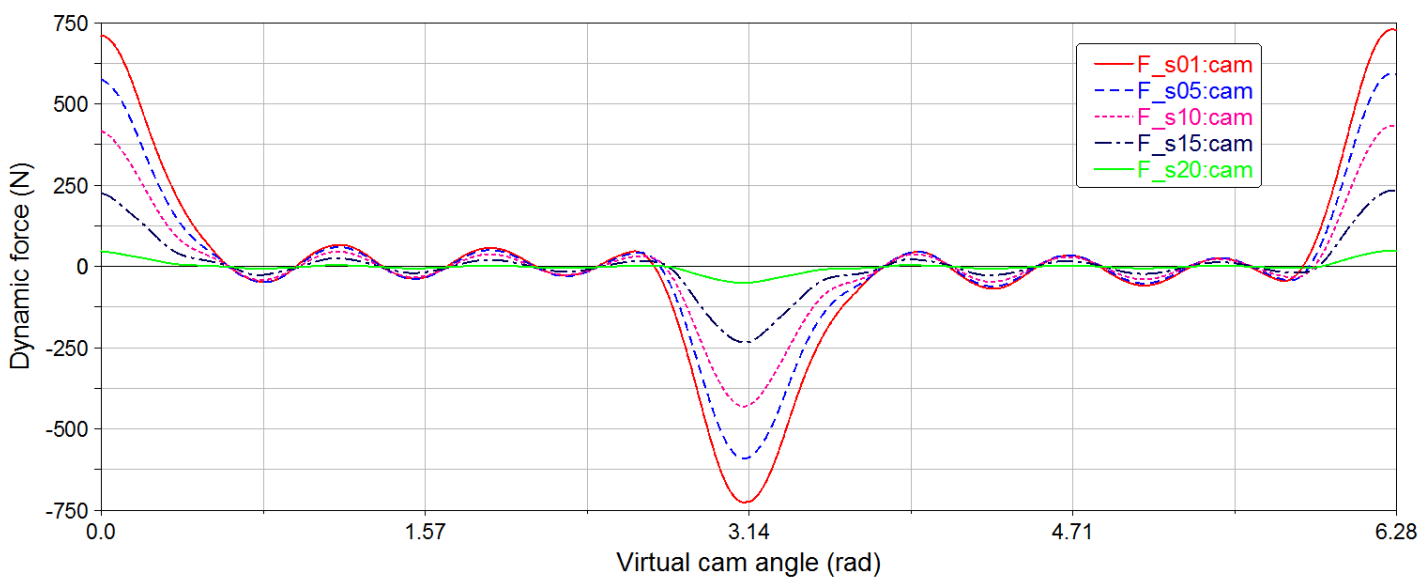

Figure 5.Dynamic force characteristic over the rod length with respect to virtual cam angle (the model with 20 sections without magnets, traversing speed 200 RPM). 
force characteristic on selected model elements. According to the theory, the maximal force is exerted in the reversal point areas reflecting the deceleration and acceleration of the rod. Over the length of the rod, the maximal force is present at the first element. The elements displacement characteristics with respect to the cam angle are shown in figure 6. Apart from longitudinal vibration of the rod, the rod deformation in the reversal areas of the motion is noticeable. The traversing movement precision is evaluated by the parameter $\Delta S$. This parameter describes the difference in the theoretical stroke that is set on the first section and the rod displacement. From figure 6 it is evident, that this value increases from the first to latter element. The maximal value is therefore at the last section. As the principal evaluation parameters of dynamic analysis, the maximal force and the maximal difference $\Delta S$ max over the rod length were chosen.

In figure 7, the overall results of the dynamic analyses are shown. The results of the original model with 20 sections are labelled sim-s20/Mg0. The maximal dynamic force in figure $7 \mathrm{a}$ increases from $100 \mathrm{~N}$ at the speed $50 \mathrm{RPM}$ up to $1180 \mathrm{~N}$ at the $250 R P M$. In figure $7 \mathrm{~b}$, the maximal displacement difference $\triangle S m a x$ is shown. It starts at the negligible value, which is caused mainly by the friction resistance, and increases up to $4.5 \mathrm{~mm}$ at the maximal simulated speed.
Results of the mathematical model have been experimentally verified by measuring the selected quantities on the full length testing rig. The testing rig consists of rod with 20 sections length and traversing mechanism with the control unit. For the force measurement, the HBM U9B load cell for tensile and compressive forces up to $5 \mathrm{kN}$ was used. It was implemented in-between the first rod and the mechanism output part. The displacement was measured at three points, in the middle of the $1^{\text {st }}, 10^{\text {th }}$ and $20^{\text {th }}$ section of the rod. For this purpose the inductive displacement transducers HBM WA with the measuring range $200 \mathrm{~mm}$ were used. The mass of measuring apparatus was included into the mathematical model of the traversing rod. The measured data were transferred from the HBM DAQ unit and processed using the Matlab software. The measurement was carried out at the velocity range 120-250 RPM. Results are shown in figure 7 under the name mea-s20/Mg0.

Both maximal force and $\Delta S \max$ correspond to results from simulation. The difference in the force at the maximal speed may be related to the effect of longitudinal vibration in the model due to its ideal joints and vibration distribution. This phenomena might be reduced on the testing rig due to additional misalignments, etc. The correspondence of analysed and measured quantities verifies the mathematical model description and appropriate material parameters settings.

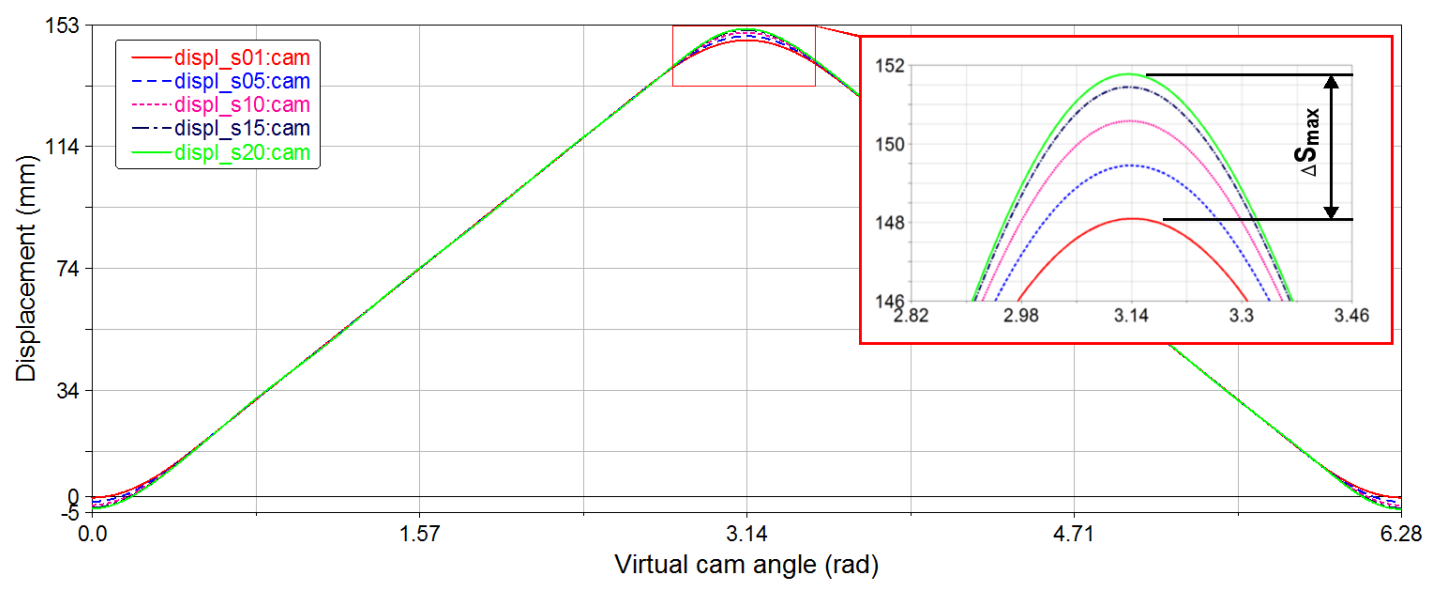

Figure 6.Displacement of elements over the rod length with respect to virtual cam angle. Definition of the $\Delta \mathrm{S}_{\max }$ (the model with 20 sections without magnets, traversing speed 200 RPM).
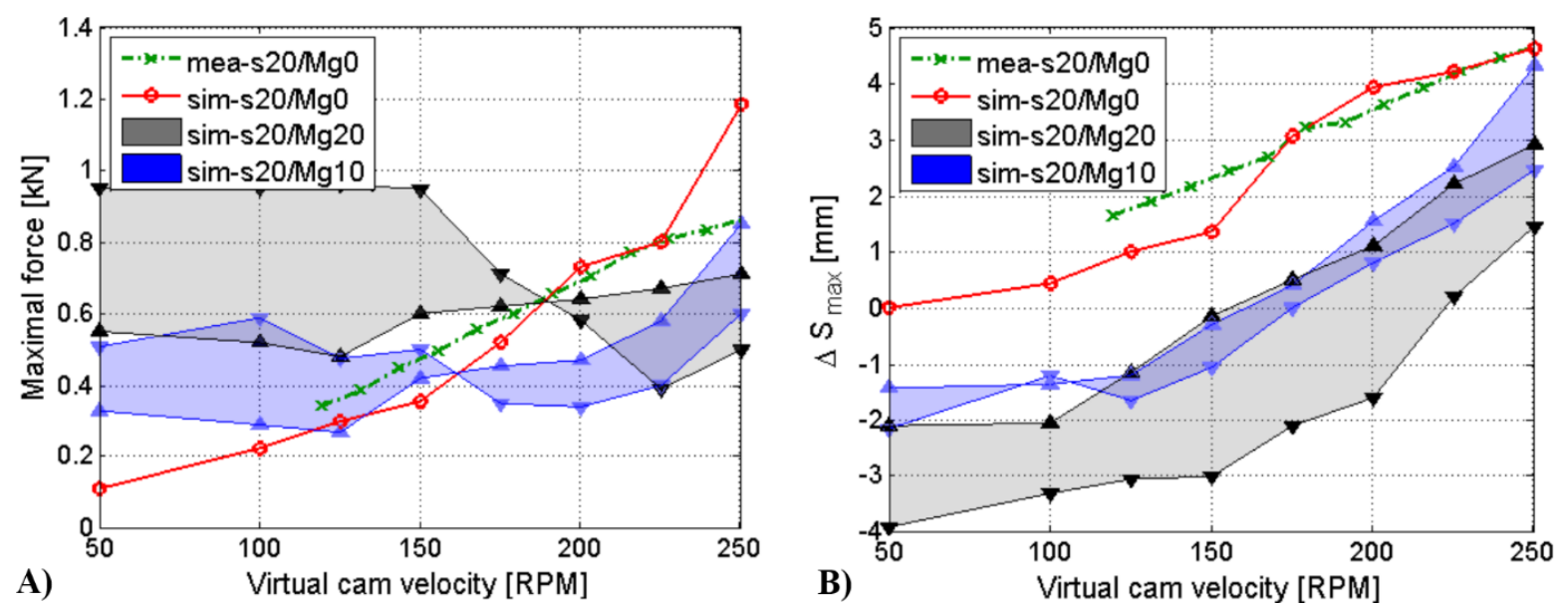

Figure 7. Results of the measurement and simulation of the system with 20-sections. A) Maximal force exerted on the traversing rod. B) Maximal displacement difference $\Delta S$ max over the traversing rod length. (Note: the reversal point shifting is described by the colour-filled area with marked boundaries, where $\boldsymbol{\Delta}$ describes the $\mathrm{I}_{\mathrm{RP}}$ reversal point and $\boldsymbol{\nabla}$ describes the $\mathrm{O}_{\mathrm{RP}}$ reversal point). 
Based on the correspondence of simulation and experiment results of the original model, the modified model was used and analysed. In order to simulate the new system behaviour with the reversal point shifting, the analysis was performed for both $I R P$ and ORP conditions (see figure1). The variable type of reversal point shifting, where $O R P-I R P=4 \mathrm{~mm}$ was used in analyses. Results from analyses are distinguished by marked boundaries, where the $\boldsymbol{\Delta}$ marker describes the results for the $I R P$ and the $\boldsymbol{\nabla}$ marker is related to the ORP. Furthermore, all considered stages of reversal point shifting are expected to fit inside the marked field.

Firstly, the configuration with 20 accumulators on 20-sectionlong model was analysed. Results from these simulations are shown in figure 7 under the name sim-s20/Mg20. High maximal forces and the significant deformation of $\triangle S$ max under IRP and $O R P$ suggest, that the overall force applied by accumulators, resp. the number of accumulators in the system, is of excessive capacity. The second studied configuration is therefore focused on the model with 10 accumulators only. In this configuration, the accumulators are placed into the even sections only and the results of this analysis are shown in figure 7 under the name sim-s20/Mg10.

\section{High-performance system analysis}

The presented system with magnetic-mechanical accumulators has been further analysed in configuration with 30-sectionlong model. For this purpose, the mathematical model of traversing rod was extended to describe the required system appropriately. The dynamic analyses were carried out for the configuration without accumulators (s30/Mg0) and with 15 accumulators (s30/Mg15) introduced to the odd sections of the model only. Analyses results are sown in figures $8 a$ and $8 b$.

\section{Results and discussion}

Both analysis and measurement results of the original model indicate the gradual increase of maximal force and $\Delta S$ max with the rising traversing speed. At the maximum speed, the simulation results show the maximal force $1180 \mathrm{~N}$, while the evaluated measurement results prove to be $880 \mathrm{~N}$. Results of the $\Delta S$ max increase gradually up to $4.5 \mathrm{~mm}$.

Results of the modified model can be divided into three distinguished operational stages. The first stage reflects the system operation at low speed and is characterized by excess force applied to the system from accumulators. This causes the rod deformation and significant force difference between the IRP and the $O R P$, whereas the maximal force is present at the ORP. The second stage is characterised by the minimal differences in the maximal force and $\triangle S$ max between the IRP and the ORP. This stage represents the optimal traversing speed for the analysed configuration of the system. The third stage reflects the high speed characterized by excessive dynamic forces and increasing rod deformation. The maximal force is here present at the IRP. However, while the $\triangle S$ max increases gradually, its difference between the IRP and ORP remains approximately constant. The difference of $\triangle S$ max between the IRP and ORP reflects the reversal point shift reduction. The difference of $2,9 \mathrm{~mm}$ (sim-s20/Mg20 at 150 RPM in figure $7 \mathrm{~b}$ ) means, the required reversal point shift of $4 \mathrm{~mm}$ to be reduced to $1,1 \mathrm{~mm}$. From the presented simulations results in figure 7 , the improvement of the analysed system with sims $20 / M g 10$ is evident. Within the range of analysed velocities (50-250 RPM), the maximal force does not exceed $840 \mathrm{~N}$ and within the velocity range (50-225 RPM) the force is below $600 \mathrm{~N}$. In this speed range, the $\Delta S$ max remains below $2,5 \mathrm{~mm}$ and its difference does not exceed the value of $1 \mathrm{~mm}$.

Analysis results of a high-performance system with 30 sections (s30/Mg0) show, that the maximal force increases up to $1200 \mathrm{~N}$ and the $\Delta$ Smax gradually rises up to $8 \mathrm{~mm}$. Results of the configuration with incorporated accumulators reflect the three stages (as mentioned above), while the optimal stage appears at the speed of approx. 150 RPM. The maximal force is below $750 \mathrm{~N}$ and within the analysed velocity range $\triangle S$ max is below $4 \mathrm{~mm}$. Furthermore, within the expected operational velocity range from 150 to $175 R P M$ this value does not exceed $2 \mathrm{~mm}$. The overall difference in $\triangle S$ max between the IRP and ORP is consistently below the value $2 \mathrm{~mm}$.
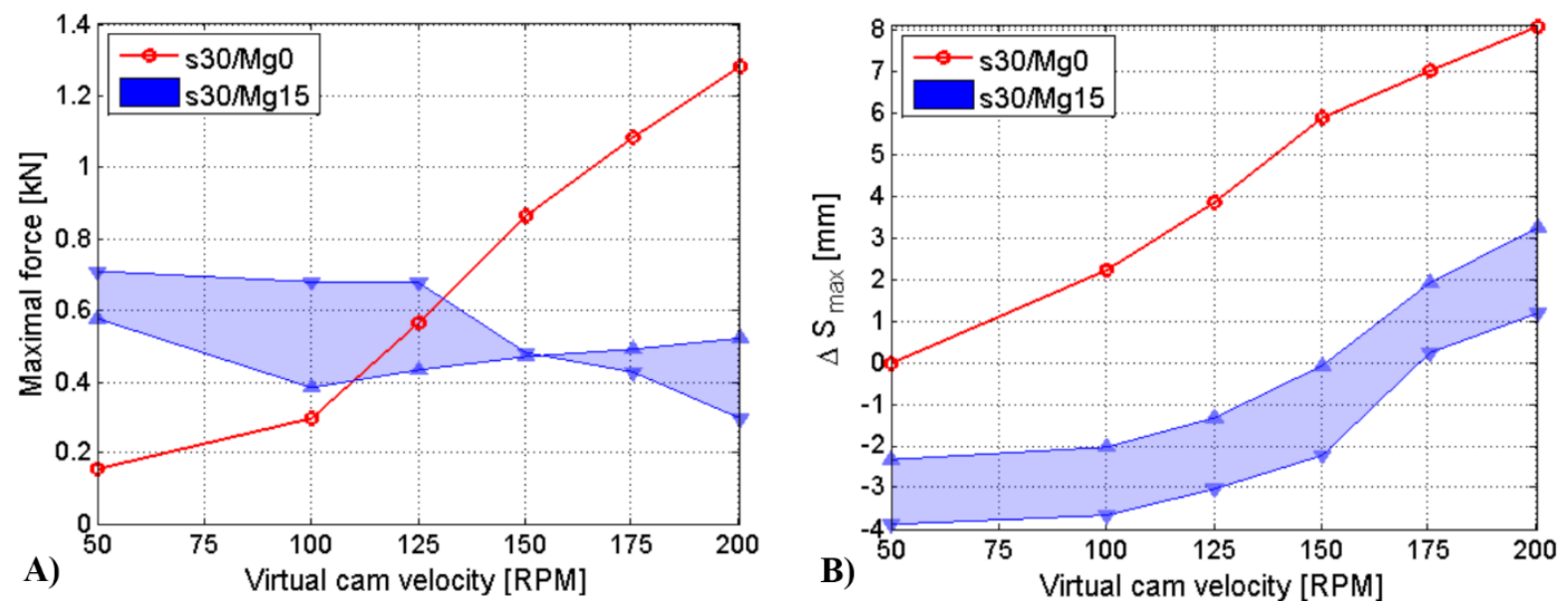

Figure 8.Results of the measurement and simulation of the system with 30-sections. A) Maximal force exerted on the traversing rod. B) Maximal displacement difference $\Delta S$ max over the traversing rod length. (Note: the reversal point shifting is described by the colour-filled area with marked boundaries, where $\boldsymbol{\Delta}$ describes the $\mathrm{I}_{\mathrm{RP}}$ reversal point and $\boldsymbol{\nabla}$ describes the $\mathrm{O}_{\mathrm{RP}}$ reversal point). 


\section{Conclusion}

In the presented paper, the central traversing system of the rotor spinning machines was described, analysed and tested. The contribution of the new magnetic-mechanical system to the dynamic force reduction of the traversing system with the rectilinear motion with variable stroke was demonstrated.

Simulation results of the original model showed the system reaches the maximal allowed tensile strength of the rod at the maximal analysed speed. In this configuration, the maximal difference in reversal point positions $\Delta S$ max increased considerably. Analysis results of the high-performance system with 30 sections proved, the maximal operational speed for such system with commonly used traversing rod to be 160 RPM. At the higher operational speeds the maximal forces exceeded the rod allowed tensile strength. The $\Delta S \max$ in this configuration also exceeded abnormally and therefore cannot meet the output quality standards.

Results of the system configurations with incorporated accumulators proved the positive impact on the reduction of the force. The allowable tensile strength of the rod was not exceeded within the analysed speed range. The potential for the modern machines is evident as the number of implemented accumulators can be extended according to required range of operational speeds or number of sections.

Regarding the traversing movement precision, a new phenomenon of the reversal point shift reduction was observed. This may affect the bobbin structure. However, the results of the system configuration with only 10 accumulators proved the maximal reversal point reduction to be within $1 \mathrm{~mm}$ only. This showed that further optimisation in a number and distribution of accumulators over the studied traversing system can be used to meet optimal traversing parameters. The developed mathematical model with programmable material parameters represents a useful tool for such a purpose.

Thanks to its design and more affordable magnetic elements, the presented system represents effective solution for both, the current and the modern high-performance rotor spinning machine in central configuration.

\section{ACKNOWLEDGEMENTS}

This publication was written at the Technical University of Liberec as part of the project 'Research of the structures and the processes of textile and single-purpose machines' with the support of the Specific University Research Grant, as provided by the Ministry of Education, Youth and Sports of the Czech Republic in the year 2015.

This work was also supported by the project FR-TI3/047 Mechatronics and Spinning Machinery.

\section{References}

[1] Valtera, J., Beran, J.: Analysis of vibration of the traversing rod on the rotor spinning machine. 1st International Summer School on Mechatronic Systems: Transfer of Innovation to the Interdisciplinary Teaching of Mechatronics for the Advanced Technology Needs, 27th September - 02nd October 2009, Wisla, s. 389 - 402. Poland. ISBN 978-8360691-56-4.

[2] Valtera, J., Beran, J. 2014. Magnetic-mechanical Accumulator of Kinetic Energy. Autex Research Journal, Volume 14, Issue 1, Pages 1-6, ISSN (Print) 1470-9589, DOI: 10.2478/v10304-012-0038 0, March 2014.

[3] Valtera, J. Optimization of the traversing rod system of rotor spinning machines. Liberec, 2013. Pp100. Doctoral thesis at The Technical University of Liberec, Czech Republic. (in Czech).

[4] Foune, F., et al. The Influence of Magnetic Accumulators Equipped with Permanent Magnets on the Drive of a Mechanical System with the Periodical Reciprocating Movement and Its Behaviour. In Vibration Problems ICOVP 2011: Proceedings of the 10th international Conference of Vibration Problems, 2011 Prague. NÁPRSTEK, J., et al. London. SPRINGER, 2011. pp. 101-106. (Springer Proceeding in Physics 139, ISBN 978 94-007-2068-8).

[5] RIKIPEDIA [on-line], URL:<http://www.rieter.com/en/ rikipedia/navelements/mainpage/> [cit. 2015 10-02].

[6] Hubálek, M.: Dynamic Analysis of the Distribution Mechanism, Proceedings of IX. International Conference on the Theory of Machines and Mechanism, 363-368, Technical University of Liberec, Liberec, (2004).

[7] Beran, J., Valtera, J.: Dynamics Analysis of the Distribution Rod on the Rotor Spinning Machine. The XIII-th Romanian Textile And Leather Conference CORTEP 2007, Iasi, "Gh. Asachi" Technical University, Faculty of Textile and Leather Engineering, 2007. ISSN 1582-6392.

[8] SAVIO. Automatic Rotor Spinning Frame FLEXIROTORS3000. [on-line], URL:< http://www. saviotechnologies.com/savio/en/Products/Rotor-SpinningFrame/Pages/Flexy-Rotors-3000.aspx > [cit.2015-10-02].

[9] Žabka, P., Valtera, J., Beran, J.: Dynamic Properties of Traversing Rod. In Advances in Mechanisms Design: Proceedings of TMM 2012, Liberec. CECARELLI, M., et al. London. SPRINGER, 2012. pp. 469-474. (Springer Mechanisms and Machine Science, Volume 8, ISBN 97894-007-5124-8.

[10] Kumar, A., Beran, J. The Traversing Mechanism of Yarn for a Group of Winding Units. In Young Researchers 2010: Proceedings of the 4th. International conference of university students within the Euroregion Nisa. 2010 Jelenia Góra. Unvwersytet Ekonomiczny we Wroclawiju, 2010. ISBN 978-83-617-19-86-1.

[11] Beran, J., Valtera, J., Žabka, P.: New Trends in Yarn Distribution Systems on Spinning Machines. The 15th International Conference STRUTEX 2008: Research Centre Textile II. Liberec, Technical University of Liberec and Czech Section of Textile Institute Manchester, Faculty of Textile Engineering, 2008. ISBN 978-80-7372-418-4. pp. 579-584. 
[12] SCHLAFHORST-SAURER. Autocoro 8 The High-tech Revolution in Rotor Spinning. [on-line], URL:<http:// schlafhorst. saurer.com > [cit.2015-10-02].

[13] Butschko, S. Cross-winding Device for a Textile Machine Which Produces Cross-wound Bobbins. OERLIKON TEXTILE GMBH \& CO KG, Butschko, S. 2008. Patent. WO2008058605 (A1).

[14] Foune, F., et al. Device for Traversing of Yarn Wound-up on the Bobbin. VUTS a.s., RIETER CZ s.r.o. European patent office: 2012 Patentový spis. EP2562112 (A1).
[15] Beran, J., Valtera, J.: Method and device for yarn distribution on textile machines. TECHNICKÁ UNIVERZITA V LIBERCI, RIETER CZ s.r.o. UPV 2011 Patent 303167.

[16] Valtera, J., Beran, J., Mathematical model of the long traversing rod with a discrete flexible link utilization, In: Proceedings of Transfer 2011 [CD-ROM], Trencin, Slovakia, November 23-24, 2011, ISBN:978-80-8075-454-9.

[17] ADAMS / Solver help - Adams 2014; Product Info \& Docs / Solver_2014 [on-line], URL:<http://simcompanion. mscsoftware.com/infocenter/> [cit. 2015-19-05]. 